\title{
Predictive Map of Geoelectric Sections of North and South America
}

\author{
Yu. B. Bashkuev, V. R. Advokatov, L. Kh. Angarkhaeva* \\ Institute of Physical Materials Science of the Siberian Branch of the Russian Academy of Sciences, Ulan-Ude, 670047, Russia \\ *Corresponding Author: ludang@rambler.ru
}

Copyright (C) 2013 Horizon Research Publishing All rights reserved.

\begin{abstract}
Predictive maps of geoelectric sections (GES) of North and South America are constructed. The GES maps are capable of increasing the accuracy of electromagnetic field calculations in the VLF-LF-MF ranges of radio waves by 1.5-3 times as compared to the Morgan-Maxwell map. The studies of electrical properties of layered media by combined radio and geophysical methods in a variety of natural and geological conditions, and the proposed method of GES mapping have resulted in the construction of a new generation of maps showing the electrical properties of the underlying medium that account for the layered structure of the crust and have no analogues in the world. Predictive geoelectric mapping of all the continents of the globe is completed.
\end{abstract}

Keywords Rocks, Geoelectric sections, Predictive map, Conductivity

\section{Introduction}

Research on the electrical properties of the Earth and the Earth's surface covers (woods, rivers, lakes, seas, and glaciers) is of major practical significance because many electromagnetic systems are located on the Earth's surface and are electrically connected with the Earth. The efficiency of telecommunications and energy systems depends substantially on the accuracy of our knowledge of the electrical characteristics of the underlying medium and their spatial and temporal variations.

The electrical properties of rocks depend on many factors, such as the lithological and mechanical compositions, water content, temperature, and groundwater mineralization; therefore, it unlikely to evaluate these parameters by theoretical calculations. They can be determined rather accurately by means of special in situ measurements. A convenient model of the underlying medium at a local point is the geoelectric section (GES) differentiated in terms of the electrical resistivity $\rho_{j}\left(\rho_{j}=1 / \sigma_{j}\right)$, permittivity $\varepsilon_{j}$, and thickness $h_{j}$ of each layer [1]. The underlying medium to a depth of 100-150 m usually consists of two to four layers with different values of $\rho_{j}$ and $\varepsilon_{j}$.

The effect of the underlying medium on the electromagnetic field is usually incorporated through the complex value of the surface impedance $\delta$, which is defined by the electrical parameters and the structure of the layered medium. Predictive maps and schemes of the distribution of various GES types over the surface form a basis for constructing frequency-dependent maps of the surface impedance, which enable the calculation of VLF-LF-MF fields. Forests, ice cover, and the seasonal freezing-thawing layer are taken into account in GES maps by introducing an additional semiconducting layer. The method of constructing GES maps is based on the relationship between the electrical properties of rocks and their lithology and geocryological conditions [2]. In addition, various geological maps, as well as extensive vertical electrical soundings (VESs), are used.

Conductivity or GES maps of continents and countries constructed on various scales according to certain criteria can be regarded as initial information for models of the electrical condition of the underlying medium. Well known are the conductivity maps of continents [3], the United States, Canada, Norway and other countries [4-6], which systematize basic data on the electrical properties of rocks in terms of a homogeneous model of the medium.

The conductivity map of the world [3] often fails to meet practical requirements because it is constructed using the model of a homogeneous underlying medium. Experimental testing of Morgan and Maxwell's map for some Siberian regions showed that the $\rho$ values on this map are, as a rule, overestimated by factors of 2-3 and more. Significant divergences with measurements are observed in some other areas as well, with electrical boundaries not coinciding with real electrical and geological boundaries. This calls for the construction of a new map of geoelectric structures of continents.

\section{Geoelectric Mapping Method}


An essential feature of the geoelectric mapping is a close relationship between physical and geological-physiographic approaches to the examination of the upper crust. In studies of electrical properties of rocks [7-9], the primary role in the identification of individual GES types is attributed to geological, climatic, and geographic factors, and regular patterns in the formation of the upper GES are closely related to the surface conditions, physical properties, structure, and composition of rocks. The construction of a predictive map of GES parameters is meant here as the determination of the surface distribution of various GES types and the estimation of the resistivity $\rho_{j}$ and thickness $h_{j}$ of individual layers of the section throughout the mapped area on the basis of a limited amount of filed geological data or measurement results. The problem reduces to the determination of the GES type (e.g., $\rho_{1}>\rho_{2}$ or $\rho_{1}<\rho_{2}$ ) of a homogeneous region and its boundaries and to the statistical estimation of the parameters $\rho_{j}$ and $h_{j}$. The methodology of geoelectric mapping is based on the following premises:

(1) there exists a correlation of the resistivity (conductivity) with lithological, hydrogeological, and frost parameters of rocks, their structure, and the GES spatial variation pattern;

(2) the resistivity of a particular rock type is a random quantity with a lognormal distribution;

(3) the boundaries of regions with identical electrical properties coincide with geological boundaries, and the rock sequence is consistent;

(4) prediction is based on geological maps of bedrock and Quaternary deposits and filed VES and drilling logs;

(5) the GES within the skin layer in the VLF range ( $f=10$ $\mathrm{kHz}$ ) is generally represented as a two- to four-layer structure; and

(6) in regions of relatively simple geological structure, prediction of GES parameters with accuracy sufficient for practical purposes GES should be based on studying key sites (territory analogues) with the subsequent extrapolation of their characteristics to geologically similar areas.

These premises are taken as guiding principles for constructing predictive geoelectric maps, showing the distribution, interrelations, and characteristics of regions with different electrical properties. The GES mapping of regions that are accessible to study is conducted in two stages. First, filed geological and geophysical data are used to construct a preliminary predictive GES map, which is checked and refined at the second stage on the basis of field measurements. Both electrical properties of homogeneous regions and their boundaries are refined. The preliminary predictive map of the first stage substantially reduces the amount of fieldwork.

The method of mapping GES parameters included a complex of radio electromagnetic profiling and sounding, vertical electrical sounding (VES), and radio comparison investigations. The profiling data enabled a more rational arrangement of key sounding sites. As a result, the required number of sounding sites decreased and the reliability of the maps increased [10]. The key sites were studied by the radio electromagnetic sounding method. The number of sounding sites within a monotypic geological rock mass depended, at a given accuracy, on the dispersion of the measured values of $|\delta|$ and $\varphi_{\delta}$.

The prediction of GES parameters is most successful in platform regions ( $\approx 70 \%$ of land), which are characterized by horizontally layered bedding of rocks with stable physical properties. The GES mapping of orogenic areas and shields with thin Quaternary deposits requires the application of the radio electromagnetic sounding method with a large number of sites, because the VES sounding in these regions is often complicated due to the mismatch between real and interpretational models. Such regions were mapped by the areal airborne radio electromagnetic profiling surveying [11]. The extensive using of VES data in the geoelectric mapping was favored by reasonable agreement between values of the impedance modulus and phase obtained by direct radio electromagnetic sounding measurements and VES-derived calculations, as well as by the absence of frequency dispersion of the conductivity of thawed rocks in a frequency range of up to $5 \mathrm{MHz}$. The convergence of the VES and radio electromagnetic sounding results confirms the validity of combining these methods for investigating the electrical parameters of the layered underlying medium in the VLF-LF-MF ranges of electromagnetic waves. As a result, it is possible to use numerous filed VES data, thereby significantly accelerating GES prediction within large regions and reducing their cost $[1,2,11]$.

Information on GES maps is expressed in codes defining the resistivities $\rho_{j}$ and thicknesses $h_{j}$ of layers on a logarithmically uniform scale. The logarithm of the discretization interval for $\rho_{j}$ and $h_{j}$ is 0.333 . Denoting the step number by $N$ and using the formulas

$$
\begin{gathered}
\left.\rho_{j}=10^{0.333(N-0.5)} \text { (in } \Omega \mathrm{m}\right), N=0-15, \\
h_{j}=10^{0.333(N-3.5)} \text { (in m), } N=1-15,
\end{gathered}
$$

we calculated the median values of $\rho_{j}$ and $h_{j}$ (Table 1 ).

Table 1. The scale of $\rho_{j}$ and $h_{j}$ parameters of geoelectric sections

\begin{tabular}{|c|c|c|}
\hline Step number & $\begin{array}{c}\text { Median value } \\
\rho_{j}, \Omega \mathrm{m}\end{array}$ & $\begin{array}{c}\text { Median value } \\
h_{j}, \mathrm{~m}\end{array}$ \\
\hline 0 & 0.68 & - \\
\hline 1 & 1.47 & 0.147 \\
\hline 2 & 3.16 & 0.316 \\
\hline 3 & 6.8 & 0.68 \\
\hline$\ldots$ & $\ldots$ & $\ldots$ \\
\hline 10 & 1470 & 147 \\
\hline 11 & 3160 & 316 \\
\hline
\end{tabular}

The map codes (e.g., 8.7.7 ...) signify the following: the first number denotes the step on the resistivity scale for the first layer (defining its $\rho_{1}$ value); the second number denotes the step on the layer thickness scale $\left(h_{1}\right)$; the third and subsequent numbers denote the same parameters for the second and subsequent layers. The permittivity of the layers was set at $\varepsilon_{j}=10$. 


\section{Main Results and Discussion}

The geoelectric structure model of the upper crust assuming the presence of electrically homogeneous layers separated by steeply dipping boundaries is a relatively rough approximation of the geological reality. However, the cognitive possibilities of this model have not yet been exhausted because the characterization of large-area zones (a few hundreds of kilometers in size) determining the major features of the geoelectric structure can be reduced to the estimation of an average geoelectric section as a first approximation.

The subject of our study, the upper crust of North and South America, is characterized by a significant spatial inhomogeneity of its electrical properties. The vast area of the territory and a very wide range of geoelectric conditions required combined investigations involving scientific analysis and generalization of both published and filed data, analytical and numerical calculations.

The initial data when constructing the predictive GES maps are, the geological 1:15000 000 map of continents [12], archival and published materials of geophysical researches (mainly by VES method). When preparing the GES maps, archival geophysical materials of industrial geological association "Abroad Geology" are widely used. These maps incorporated the Institute of Physical Materials Science data derived from geoelectric investigations in 1971-2012.

Figures 1,2 present the predictive GES maps of North and South America. The map of North America, covering an area of $24250000 \mathrm{~km}^{2}$, contains 22 types of two- to three-layer geoelectric sections; the map of South America, covering an area of $17800000 \mathrm{~km}^{2}$, contains 16 GES types.

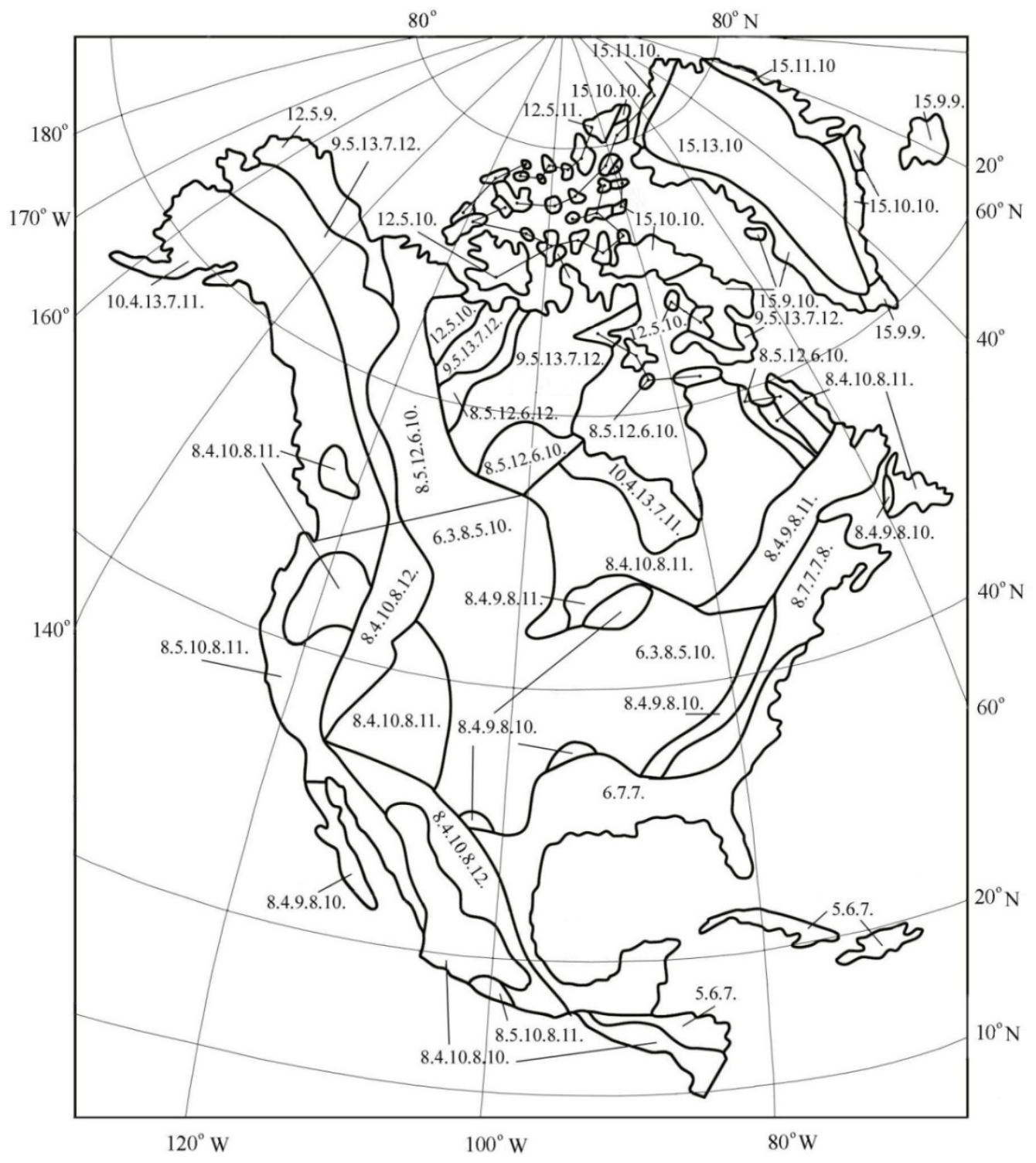

Figure 1. The predictive map of geoelectric sections of North America 


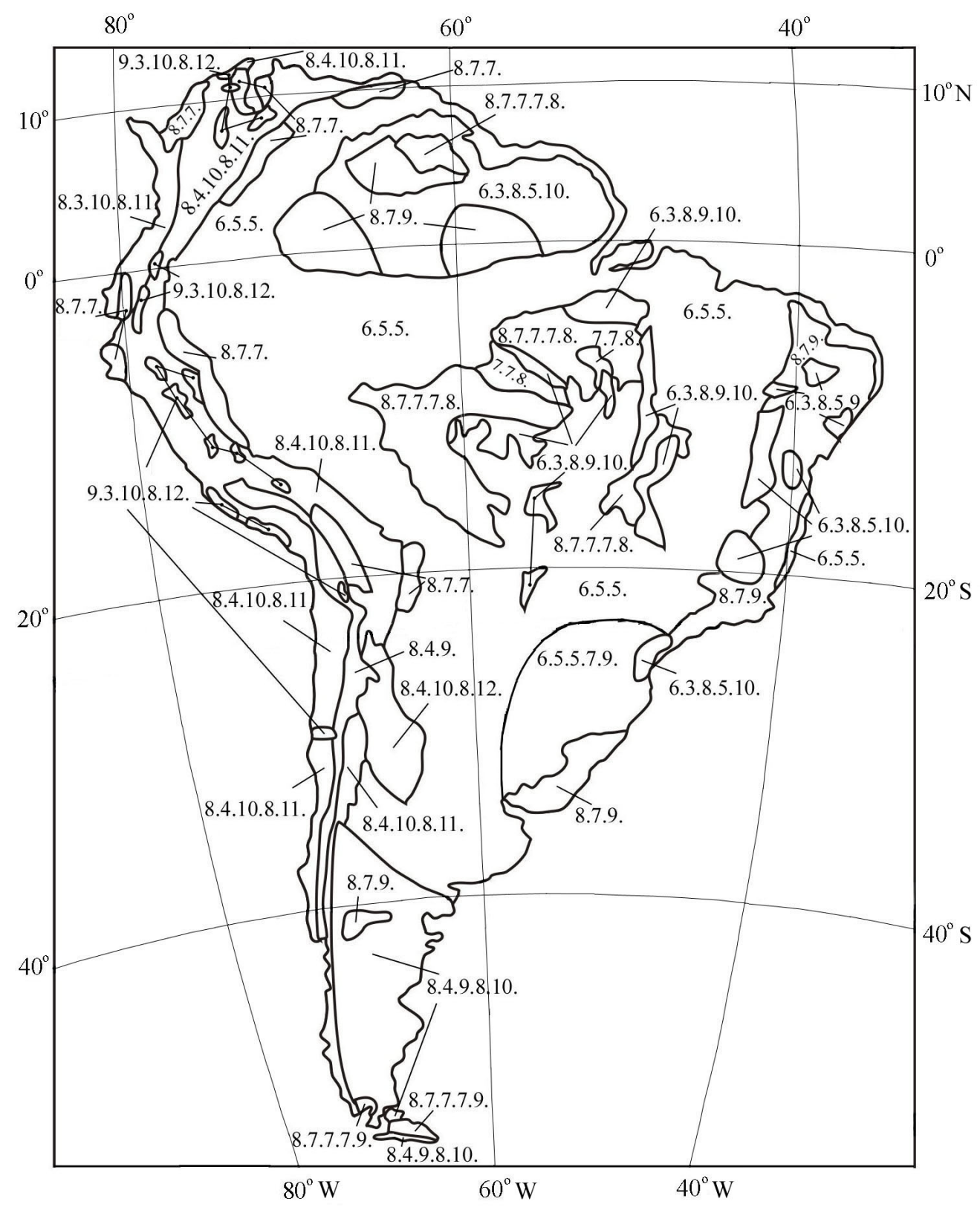

Figure 2. The predictive map of geoelectric sections of South America

Figure 3 illustrates the frequency dependences of the modulus $|\delta|$ and phase $\varphi_{\delta}^{\circ}$ of the surface impedance for the most widespread GES types in North and South America. The surface impedance of an $n$-layer medium was calculated with the formula $\delta^{(n)}=\delta_{1} Q^{(n)}$, which is convenient for numerical calculations [1]. Here, $\delta_{1}$ is the surface impedance of a homogeneous medium with the parameters of the first layer, and $Q^{(n)}=F\left(f, \rho_{j}, \varepsilon_{j}, h_{j}\right)$ is the correction factor accounting for the underlying crustal layers. Analysis of the impedance modulus $|\delta|$ and phase $\varphi_{\delta}{ }^{\circ}$ shown in the GES map reveals a significant range of their variation. For example, the values of $|\delta|$ at a frequency of $10 \mathrm{kHz}$ vary from 0.0044 to 0.058 , and the impedance phase, from $-37^{\circ}$ to $-48^{\circ}$; the respective variation ranges at $1000 \mathrm{kHz}$ are 0.061 to 0.32 and $-2^{\circ}$ to $-49^{\circ}$.
With account taken of the topography and woods, the GES maps make it possible to determine such an important characteristic of the electromagnetic wave process as the function of the $W$ field attenuation within a wide range of electromagnetic waves. The $W$ values in the model of a multisection impedance propagation path are determined by solving numerically the Hufford integral equation [13]. The accuracy of predicting the electromagnetic field attenuation is $\pm(15-30) \%[2,11]$.

The GES maps of North and South America were constructed from dry season data. The conditions in the upper GES layer vary over the annual cycle, producing related variations in the distribution patterns of the temperature, structure, water content, salt composition, and water phase state in rocks. Equatorial and subtropical regions 
are characterized by an irregular precipitation distribution over the year. The variability in the electrical state of GESs in these regions is due mainly to mass exchange (water and ion transport). The model of a layered medium with climate-dependent properties and structure should account for the seasonal variations in the impedance of the boundary surface and the near-surface electromagnetic field.
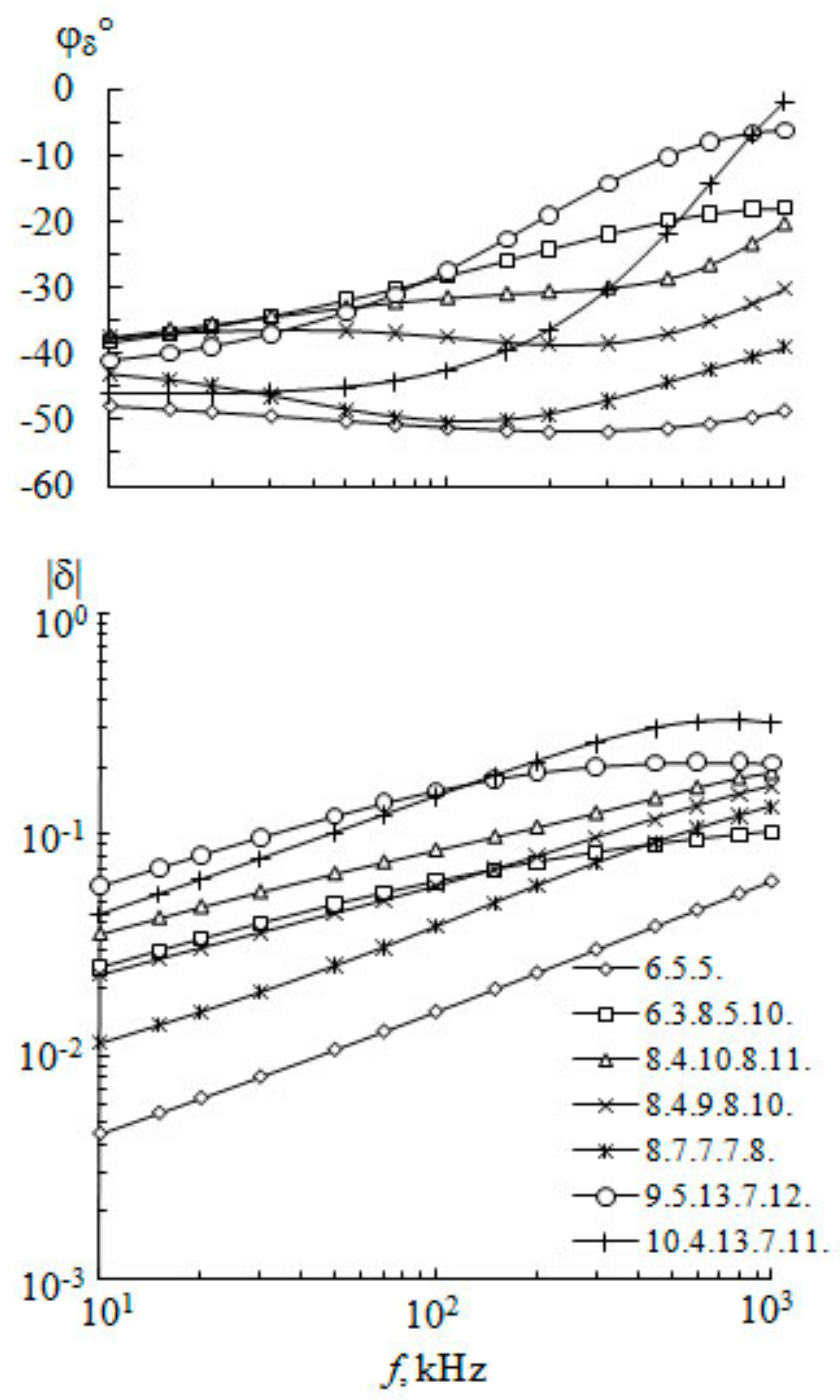

Figure 3. Frequency dependences of the surface impedance modulus $|\delta|$ and phase $\varphi_{\delta}{ }^{\circ}$ for the most widespread common GES types in North and South America

The GES maps provide a generalized image of the real geological situation, and its reliability depends on the density of observations and their generalization in accordance with the chosen scale. (In cartography, the term "generalization" refers to the selection of the most essential features and their generalization (sensu lato) in accordance with the purpose, subject, and scale of the map.) Geoelectric maps have their specific scale-dependent features. In large-scale maps, the geoelectric situation is usually imaged on the basis of direct measurements because in this case the number of observation sites is adequate. In small-scale mapping, one has to resort to interpolation and extrapolation of regular patterns identified at key sites.

The GES maps were constructed on the basis of a limited amount of initial data that are very irregularly distributed over surface, which precluded formal interpolation and extrapolation with the required degree of detail and accuracy. In such a situation, we used the method of analogies, which is common in the Earth sciences. Prediction of GES parameters relied on the study of geoelectric features in a particular region and detailed examination of the key and prediction areas. The work was done in the following order. The most probable values of the GES parameters $\rho_{j}$ and $h_{j}$ were determined in a key area. The similarity between the key and prediction areas suggested from their geological and geophysical characteristics allowed us to extend the $\rho_{j}$ and $h_{j}$ values obtained at the key site to the entire study area with similar properties. Results of the prediction largely depend on the adequate selection of key sites within the prediction area. The prediction accuracy is difficult to estimate rigorously. Our experience shows that reliable constraints are gained not from the statistical estimation of reliability but from the results of checking the GES parameters in adequately selected control areas. The GES parameters of key sites (individual rock types) were estimated from a limited data sample on the basis of statistical processing of the initial $\rho_{j}$ and $h_{j}$ data set. The construction of $\rho_{j}$ and $h_{j}$ histograms enabled the determination of the $\rho_{j}$ and $h_{j}$ distribution patterns and in several cases allowed us to eliminate gross errors in their estimation.

The electrical characteristics of the study regions were shown to be typical of the continental crust; they show a regular spatial variation and depend on the type of geoelectric structure (depression, massif, fault zone, etc.) and their association with a particular complex of crystalline and sedimentary rocks [11]. Geoelectric mapping also showed that the transition from one geological section to another is accompanied by a change in electrical properties, i.e., geoelectric and geological boundaries usually coincide [11]. In some of the areas mapped, the problem of the boundaries of the inferred regions and the values of $\rho_{j}$ and $h_{j}$ ascribed to them is debatable; therefore, we restrained from mapping the difference in $\rho_{j}$ and $h_{j}$ between these regions. The majority of the geoelectric boundaries coincides with the geological boundaries or faults and is reliably delineated; some boundaries are indistinct and drawn tentatively because they fall within wide zones of gradual transition of $\rho_{j}$ and $h_{j}$ values. Thus, the probability of correct estimation of geoelectric parameters was raised both through the combined use of all available geophysical data and by utilizing the electrical characteristics of analogous territories and key sites.

As regards the amount of available empirical data, our GES maps of North and South America on a scale of 1:15 000000 fall under the category of schematic or predictive maps. They share all the features inherent in small-scale maps. When working with them, one should keep in mind the resolution of small-scale maps and their inability to image some, occasionally essential, details of the geoelectric situation that are clearly visible in maps of 
larger scales. One should always consider that the contours bounding a given GES type always include areas of a different GES type.

\section{Conclusion}

The studies of electrical properties of layered media by combined radio and geophysical methods have resulted in the construction of a new generation of maps showing the electrical properties of the underlying medium that account for the layered structure of the crust and have no analogues in other countries [14-16]. These maps reflect the areal distribution of various GES types, with the electrical resistivity $\rho_{j}$ and thickness $h_{j}$ specified for each layer.

The GES maps comprehensively characterize the regional patterns of spatial distribution of areas with different GES types on the continents of the Eastern and Western Hemisphere. These are general maps as regards their scale and specialized maps with respect to their content. They are the first attempt to generalize the vast and diverse empirical data and to show that their underlying premises and the method of their construction ensure an adequate reflection of the relationship between the electrical properties of the upper crust and various geological structures.

The method of GES mapping proposed in this work allowed us to elaborate a unified approach to the representation of the real geological situation in a 3-D model on a quantitative basis. Moreover, the method of predicting electrical properties of the upper crust has markedly reduced the direct costs of experiments in forested mountains, deserts and other areas that are difficult to access.

The GES maps can be useful in calculations of the generation and propagation of VLF-LF-MF electromagnetic waves; design of grounding and lightning protection for power lines, electrical installations, and communications systems; and solution of electromagnetic earthquake prediction and geological engineering problems. Taking into account the layered structure of the underlying medium, these maps are capable of increasing the accuracy of electromagnetic field calculations by $1.5-3$ times compared to the Morgan-Maxwell map.

Predictive geoelectric mapping of all the continents of the globe completed. We now need to collect all the cards together in the form of "Atlas of predictive maps of geoelectric sections of countries and continents of the globe."

\section{Acknowledgements}

This article was prepared with the partial financial support of RFBR, grant numbers 12-02-98002, 12-01-98010, and SB RAS integration project number 11.

\section{REFERENCES}

[1] Ch. T. Tsydypov, V. D. Tsydenov, Yu. B. Bashkuev. Investigation of Electric Properties of the Underlying Medium, Nauka, Novosibirsk, Russia, 1979.

[2] V. S. Dorzhiev, V. R. Advokatov, B. B. Bodiev. Geoelectric Structures of Southern Siberia and Mongolia, Nauka, Moscow, Russia, 1987.

[3] R. Morgan, E. Maxwell. Omega Navigation System Conductivity Map, Office of Naval Research, Washington, 1965.

[4] H. Fine. An Effective Ground Conductivity Map for Continental United States, Proc. IRE, Vol.42, No.9, 1405-1408, 1954.

[5] G. C. Ireland. A Provisional Ground Conductivity Map of Canada, Proc. IRE, Vol.49, No.11, 1674-1678, 1961.

[6] K. E. Eliassen. A Survey of Ground Conductivity and Dielectric Constant in Norway within the Frequency Range 0,2-10 Mc/s, Geophys. Publ., Vol.19, No.11, 5-30, 1957.

[7] V. S. Yakupov. Electric Conductivity and the Geoelectric Section of Frozen Rocks, Nauka, Moscow, Russia, 1968.

[8] V. P. Mel'nikov. Electrophysical Investigations of Permafrost Beds, Nauka, Novosibirsk, Russia, 1977.

[9] A. V. Veshev. Direct and Alternating Current Electric Profiling, Nedra, Leningrad, Russia, 1980.

[10] A. A. Pylaev. The State and Problems of Mapping Geoelectric Properties of the Underlying Surface in Relation to the Estimation of the Earth Wave Propagation, XIV Interdept. Seminar on the Propagation of 1-km and Longer Radio Waves, Gorki, 8-14, 1989.

[11] Yu. B. Bashkuev. Electrical Properties of Natural Layered Media, SO RAN, Novosibirsk, 1996.

[12] The Geological Map of Continents of Scale 1 : 15 000000, Aerogeology, Moscow, Russia, 1973.

[13] G. A. Hufford. An Integral Equation Approach to the Problem of Wave Propagation over an Irregular Surface, Quart. Appl. Math., Vol.9, 391-404, 1952.

[14] V. S. Dorzhiev, Yu. B. Bashkuev, Ch. T. Tsydypov, V. R. Advokatov, Yu. A. Ivoilov. The Map of Geoelectric Sections of Mongolia, Proceedings of the VII Scientific Conference on Thematic Cartography, Publishing House of the Institute of Geography of the Russian Academy of Sciences, Irkutsk, 78-81, 2002.

[15] Yu. B. Bashkuev, V. R. Advokatov, L. Kh. Angarkhaeva. Maps of Geoelectric Sections in the Eastern Hemisphere, Fizika Zemli, No.9, 87-94, 2003.

[16] Yu. B. Bashkuev, V. R. Advokatov, L. Kh. Angarkhaeva, V. S. Dorzhiev, M. Hayakawa. Maps of Geoelectric Sections of Turkey, Iran, Afghanistan, Pakistan, Korea, and Japan, Natural Hazards and Earth System Sciences, No.8, 861-868, 2008 